\title{
RUPTURE OF PERONEUS BREVIS TENDON MIMICKING GIANT CELL TUMOUR OF PERONEAL TENDONS SHEATH - CASE REPORT
}

\author{
Authors: Ana Giljanović ${ }^{1}$, Ivana Karla Franić ${ }^{1}$, Ana Gašić ${ }^{1}$, Damjan Dimnjaković ${ }^{2}$ (mentor) \\ ${ }^{1}$ School of Medicine University of Zagreb, Zagreb, Croatia \\ ${ }^{2}$ Department of orthopaedics, University Hospital Centre Zagreb \& School of Medicine, University of \\ Zagreb,Croatia
}

DOI: https://doi.org/10.26800/LV-142-supp5-8

\section{Background:}

Peroneal tendon pathology, which also includes post-traumatic tendon rupture is often overlooked cause of persistent pain and localized swelling in the lateral part of the ankle and foot. We report a case of peroneus brevis tendon rupture presenting as a palpable mass in the lateral part of the ankle, mimicking a giant cell tumour of peroneal tendons sheath.

\section{Case presentation:}

A fifty-seven-year-old male patient presented himself with localized mass and pain in the lateral part of the foot and ankle. The patient stated that he had blunt trauma to the lateral side of the right foot two years earlier. Clinical examination revealed a painful mass located $2 \mathrm{~cm}$ distal from the tip of the fibular malleolus. An MRI was conducted indicating giant cell tumour of peroneal tendon sheath as the possible diagnosis. Surgical excision of the tumour was indicated, but the intraoperative finding revealed that the mass was part of the ruptured peroneus brevis tendon. Excision of the mass and the remaining part of the ruptured tendon was performed, followed by the tenodesis of the proximal and distal part of the peroneus brevis tendon to the peroneus longus tendon. The histopathological analysis described the excised tissue as posttraumatic pseudocystic degeneration of the tendon. The postoperative period was uneventful and the patient made a full recovery.

\section{Conclusion:}

Even though MRI is a powerful imaging tool for evaluation of the peroneal tendons, the detailed medical history and examination is the key to the proper diagnosis of peroneal tendon pathology. In cases with a complete rupture of peroneus brevis tendon, tenodesis to the peroneus longus tendon is a valuable surgical option with good functional results.

Keywords: giant cell tumour, peroneal tendons, rupture 